\title{
Fixed Point Theorems for Weakly Compatible Functions using (JCLR) Property in Intuitionistic Fuzzy Metric Space
}

\author{
Jong Seo Park* \\ ${ }^{*}$ Department of Mathematic Education, Chinju National University of Education, \\ Jinju 660-756, South Korea
}

\begin{abstract}
In this paper, we give definitions for common limit in the range property of mappings and obtain common fixed point theorem for a pair of weakly compatible functions in intuitionistic fuzzy metric space using the joint common limit in the range property of mappings(shortly, (JCLR) property). Our results improve and generalize results of Chauhan et al[1].
\end{abstract}

Key Words: Fixed point, Weakly compatible function, (JCLR) Property.

\section{Introduction}

Zadeh[10] researched the concept of a fuzzy set. In 1975, George and Veeramani[2] modified the concept of fuzzy metric space introduced by Kramosil and Michalek[4] with a view to obtain a Hausdorff topology, and this has recently found very fruitful applications in quantum particle physics. In recent years, many authors have proved fixed point theorems in fuzzy metric spaces, and observed some common fixed point theorems in fuzzy metric space which improved many known results([3], [9] etc). Chauhan et al[1] introduced the notion of (JCLR) property and obtained fixed point theorem in fuzzy metric space. Park[5] studied some properties for compatible map in intuitionistic fuzzy metric space. Also, Park[6],[7] defined the intuitionistic fuzzy contraction, and some fixed point theorem using common property(E.A.) and weakly compatibility in intuitionistic fuzzy metric space.

In this paper, we obtain common fixed point theorem for a pair of weakly compatible functions using the joint common limit in the range property of mappings(shortly, (JCLR) property) in intuitionistic fuzzy metric space. Our results improve and generalize results of Chauhan et al[1].

\section{Preliminaries}

In this part, we recall some definitions, properties and known results in the intuitionistic fuzzy metric space as fol-

2000 Mathematics Subject Classification. 46S40, 47H10, 54H25. Manuscript received Nov. 30, 2012; revised Dec. 24, 2012; accepted Dec. 24, 2012.

*Corresponding Author: Jong Seo Park, parkjs@cue.ac.kr

(c) The Korean Institute of Intelligent Systems. All rights reserved. lowing :

Let us recall(see [5]) that a continuous $t$-norm is a operation $*:[0,1] \times[0,1] \rightarrow[0,1]$ which satisfies the following conditions: (a) $*$ is commutative and associative, (b)* is continuous, (c) $a * 1=a$ for all $a \in[0,1]$, (d) $a * b \leq c * d$ whenever $a \leq c$ and $b \leq d(a, b, c, d \in[0,1])$. Also, a continuous $t$-conorm is a operation $\diamond:[0,1] \times[0,1] \rightarrow[0,1]$ which satisfies the following conditions: (a) $\diamond$ is commutative and associative, (b) $\diamond$ is continuous, (c) $a \diamond 0=a$ for all $a \in[0,1],(\mathrm{d}) a \diamond b \geq c \diamond d$ whenever $a \leq c$ and $b \leq d$ $(a, b, c, d \in[0,1])$.

Definition 2.1. ([5])The 5 -tuple $(X, M, N, *, \diamond)$ is said to be an intuitionistic fuzzy metric space if $X$ is an arbitrary set, $*$ is a continuous $t$-norm, $\diamond$ is a continuous $t$-conorm and $M, N$ are fuzzy sets on $X^{2} \times(0, \infty)$ satisfying the following conditions; for all $x, y, z \in X$, such that

(a) $M(x, y, t)>0$,

(b) $M(x, y, t)=1$ if and only if $x=y$,

(c) $M(x, y, t)=M(y, x, t)$,

(d) $M(x, y, t) * M(y, z, s) \leq M(x, z, t+s)$,

(e) $M(x, y, \cdot):(0, \infty) \rightarrow(0,1]$ is continuous,

(f) $N(x, y, t)>0$,

(g) $N(x, y, t)=0$ if and only if $x=y$,

(h) $N(x, y, t)=N(y, x, t)$,

(i) $N(x, y, t) \diamond N(y, z, s) \geq N(x, z, t+s)$,

(j) $N(x, y, \cdot):(0, \infty) \rightarrow(0,1]$ is continuous.

Note that $(M, N)$ is called an intuitionistic fuzzy metric on $X$. The functions $M(x, y, t)$ and $N(x, y, t)$ denote the degree of nearness and the degree of non-nearness between $x$ and $y$ with respect to $t$, respectively.

Let $X$ be an intuitionistic fuzzy metric space. For $t>0$, the open ball $B(x, r, t)$ with center $x \in X$ and radius $0<$ 
$r<1$ is defined by

$B(x, r, t)=\{y \in X ; M(x, y, t)>1-r, N(x, y, t)<r\}$.

Now, let $X$ be an intuitionistic fuzzy metric space and $\tau$ the set of all $A \subset X$ with $x \in A$ if and only if there exist $t>0$ and $0<r<1$ such that $B(x, r, t) \subset A$. Then $\tau$ is a topology on $X$ induced by the intuitionistic fuzzy metric $M, N$.

Definition 2.2. ([8]) Let $X$ be an intuitionistic fuzzy metric space.

$M$ and $N$ are said to be continuous on $X^{2} \times(0, \infty)$ if

$$
\begin{aligned}
& \lim _{n \rightarrow \infty} M\left(x_{n}, y_{n}, t\right)=M(x, y, t), \\
& \lim _{n \rightarrow \infty} N\left(x_{n}, y_{n}, t\right)=N(x, y, t)
\end{aligned}
$$

whenever a sequence $\left\{\left(x_{n}, y_{n}, t_{n}\right)\right\}$ in $X^{2} \times(0, \infty)$ converge to a point $(x, y, t) \in X^{2} \times(0, \infty)$, that is,

$$
\begin{aligned}
& \lim _{n \rightarrow \infty} M\left(x_{n}, x, t\right)=\lim _{n \rightarrow \infty} M\left(y_{n}, y, t\right)=1, \\
& \lim _{n \rightarrow \infty} N\left(x_{n}, x, t\right)=\lim _{n \rightarrow \infty} N\left(y_{n}, y, t\right)=0
\end{aligned}
$$

and

$$
\begin{aligned}
& \lim _{n \rightarrow \infty} M\left(x, y, t_{n}\right)=M(x, y, t), \\
& \lim _{n \rightarrow \infty} N\left(x, y, t_{n}\right)=N(x, y, t) .
\end{aligned}
$$

Lemma 2.3. ([5]) Let $X$ be an intuitionistic fuzzy metric space. If there exists $k \in(0,1)$ such that

$$
M(x, y, k t) \geq M(x, y, t), \quad N(x, y, k t) \leq N(x, y, t)
$$

for all $x, y \in X$ and $t>0$, then $x=y$.

Definition 2.4. ([6]) A pair of self mappings $(f, g)$ defined on an intuitionistic fuzzy metric space $X$ is said to satisfy the property(E.A.) if there exists a sequence $\left\{x_{n}\right\} \subset X$ such that $\lim _{n \rightarrow \infty} f x_{n}=\lim _{n \rightarrow \infty} g x_{n}=z$ for some $z \in$ $X$.

Definition 2.5. ([7]) Two self mappings $f$ and $g$ on an intuitionistic fuzzy metric space $X$ are called weakly compatible(or coincidentally commuting) if they commute at their point of coincidence. That is, if $f x=g x$ for some $z \in X$, then $f g x=g f x$.

Definition 2.6. A pair $(f, g)$ of self mappings of an intuitionistic fuzzy metric space $X$ is said to satisfy the common limit in the range of $g$ property(shortly, (CLRg) property) if there exists a sequence $\left\{x_{n}\right\} \subset X$ such that $\lim _{n \rightarrow \infty} f x_{n}=\lim _{n \rightarrow \infty} g x_{n}=g u$ for some $u \in X$.
Example 2.7. Let $X$ be an intuitionistic fuzzy metric space with $X=[0, \infty)$ and

$$
\begin{aligned}
& M(x, y, t)= \begin{cases}\frac{t}{t+|x-y|} & \text { if } t>0, \\
0 & \text { if } t=0\end{cases} \\
& N(x, y, t)= \begin{cases}\frac{|x-y|}{t+|x-y|} & \text { if } t>0 \\
1 & \text { if } t=0\end{cases}
\end{aligned}
$$

for all $x, y \in X$. Define self mappings $f$ and $g$ on $X$ defined by $f(x)=\frac{x}{8}$ and $g(x)=\frac{3 x}{7}$ for all $x \in X$. If $\left\{x_{n}\right\}=$ $\left\{\frac{1}{n}\right\}_{n \in N}$ in $X$. Since $\lim _{n \rightarrow \infty} f x_{n}=\lim _{n \rightarrow \infty} g x_{n}=$ $0=g(0)$ for $0 \in X$. Therefore $f$ and $g$ satisfy the (CLRg) property.

Definition 2.8. ([1]) Two families of self mappings $\left\{f_{i}\right\}_{i=1}^{m}$ and $\left\{g_{k}\right\}_{k=1}^{n}$ are said to be pairwise commuting if

a) $f_{i} f_{j}=f_{j} f_{i}$ for all $i, j \in\{1,2, \cdots, m\}$,

b) $g_{k} g_{l}=g_{l} g_{k}$ for all $k, l \in\{1,2, \cdots, n\}$,

c) $f_{i} g_{k}=g_{k} f_{i}$ for all $i \in\{1,2, \cdots, m\}$ and $k \in$ $\{1,2, \cdots, n\}$.

Implicit relations on intuitionistic fuzzy metric spaces have been used in many articles([5], [7] etc). Let $\Psi=$ $\{\phi, \psi\}$ be implicit functions set, $I=[0,1], \phi, \psi: I^{5} \rightarrow I$ be continuous functions following conditions :

(I) $\phi$ is decreasing and $\psi$ is increasing in five variables.

(II) $\phi(t, t, t, t, t)>t$ and $\psi(t, t, t, t, t)<t$ for all $t \in$ $[0,1)$.

\section{Main Result}

Definition 3.1. Let $X$ be an intuitionistic fuzzy metric space and $f, g, u, v: X \rightarrow X$. The pair $(f, v)$ and $(u, g)$ are said to satisfy the joint common limit in the range of $v$ and $g$ property(shortly, (JCLRvg) property) if there exists a sequence $\left\{x_{n}\right\}$ and $\left\{y_{n}\right\}$ in $X$ such that

$$
\begin{aligned}
\lim _{n \rightarrow \infty} f x_{n} & =\lim _{n \rightarrow \infty} v x_{n}=\lim _{n \rightarrow \infty} u y_{n} \\
& =\lim _{n \rightarrow \infty} g y_{n}=v z=g z
\end{aligned}
$$

for some $z \in X$.

Remark 3.2. If $f=u$ and $g=v$ and $\left\{x_{n}\right\}=\left\{y_{n}\right\}$ in (1), then we get the definition of (CLRg) property.

Theorem 3.3. Let $X$ be an intuitionistic fuzzy metric space, where $*, \diamond$ are continuous $\mathrm{t}$-norm, $\mathrm{t}$-conorm and $f, g, u$ and $v$ be mappings from $X$ into itself. Further, let the pair $(f, v)$ and $(v, g)$ are weakly compatible and there 
exists a constant $k \in\left(0, \frac{1}{2}\right)$ such that

$$
\begin{aligned}
& M(f x, u y, k t) \quad \geq \phi(M(v x, g y, t), M(f x, v x, t), \\
& M(u y, g y, t), M(f x, g y, \alpha t), \\
& M(u y, v x, 2 t-\alpha t)) \text {, } \\
& N(f x, u y, k t) \leq \psi(N(v x, g y, t), N(f x, v x, t), \\
& N(u y, g y, t), N(f x, g y, \alpha t) \text {, } \\
& N(u y, v x, 2 t-\alpha t))
\end{aligned}
$$

hold for all $x, y \in X, \alpha \in(0,2), t>0$ and $\phi, \psi \in \Phi$. If $(f, v)$ and $(u, g)$ satisfy the (JCLRvg) property, then $f, g, u$ and $v$ have a unique common fixed point in $X$.

Proof. Since the pairs $(f, v)$ and $(u, g)$ satisfy the (JCLRvg) property, there exists a sequence $\left\{x_{n}\right\},\left\{y_{n}\right\} \subset$ $X$ such that $\lim _{n \rightarrow \infty} f x_{n}=\lim _{n \rightarrow \infty} v x_{n}=$ $\lim _{n \rightarrow \infty} u y_{n}=\lim _{n \rightarrow \infty} g y_{n}=v z=g z$ for some $z \in X$.

Now, we assert that $g z=u z$. Using (2), with $x=x_{n}$, $y=z$ for $\alpha=1$, we get

$$
\begin{aligned}
M\left(f x_{n}, u z, k t\right) \leq & \phi\left(M\left(v x_{n}, g z, t\right), M\left(f x_{n}, v x_{n}, t\right),\right. \\
& M(u z, g z, t), M\left(f x_{n}, g z, t\right), \\
& \left.M\left(u z, v x_{n}, t\right)\right), \\
N\left(f x_{n}, u z, k t\right) \quad \leq & \psi\left(N\left(v x_{n}, g z, t\right), N\left(f x_{n}, v x_{n}, t\right),\right. \\
& N(u z, g z, t), N\left(f x_{n}, g z, t\right), \\
& \left.N\left(u z, v x_{n}, t\right)\right) .
\end{aligned}
$$

Taking the limit as $n \rightarrow \infty$, we have

$$
\begin{array}{cl}
M(g z, u z, k t) \geq & \phi(M(g z, g z, t), M(g z, g z, t), \\
& M(u z, g z, t), M(g z, g z, t), \\
& M(u z, g z, t)), \\
N(g z, u z, k t) \leq & \psi(N(g z, g z, t), N(g z, g z, t), \\
& N(u z, g z, t), N(g z, g z, t), \\
& N(u z, g z, t)) .
\end{array}
$$

Since $\phi$ is increasing, and $\psi$ is decreasing in each of its coordinate and $\phi(t, t, t, t, t)>t$ and $\psi(t, t, t, t, t)<t$ for all $t \in[0,1)$, we get $M(g z, u z, k t) \geq M(g z, u z, t)$ and $N(g z, u z, k t) \leq N(g z, u z, t)$. By Lemma 2.3, we have $g z=u z$

Next, we show that $f z=g z$. Using (2) with $x=z$, $y=y_{n}$ for $\alpha=1$, we have

$$
\begin{aligned}
M\left(f z, u y_{n}, k t\right) \geq & \phi\left(M\left(v z, g y_{n}, t\right), M(f z, v z, t),\right. \\
& M\left(u y_{n}, g y_{n}, t\right), M\left(f z, g y_{n}, t\right), \\
& \left.M\left(u y_{n}, v z, t\right)\right), \\
N\left(f z, u y_{n}, k t\right) \leq & \psi\left(N\left(v z, g y_{n}, t\right), N(f z, v z, t),\right. \\
& N\left(u y_{n}, g y_{n}, t\right), N\left(f z, g y_{n}, t\right), \\
& \left.N\left(u y_{n}, v z, t\right)\right) .
\end{aligned}
$$

Taking the limit as $n \rightarrow \infty$, we have

$$
\begin{array}{cl}
M(f z, g z, k t) \geq & \phi(M(g z, g z, t), M(f z, g z, t), \\
& M(g z, g z, t), M(f z, g z, t), \\
& M(g z, g z, t)), \\
N(f z, g z, k t) \leq & \psi(N(g z, g z, t), N(f z, g z, t), \\
& N(g z, g z, t), N(f z, g z, t), \\
& N(g z, g z, t)) .
\end{array}
$$

Since $\phi$ is increasing, and $\psi$ is decreasing in each of its coordinate and $\phi(t, t, t, t, t)>t$ and $\psi(t, t, t, t, t)<t$ for all $t \in[0,1)$, we get $M(f z, g z, k t) \geq M(f z, g z, t)$ and $N(f z, g z, k t) \leq N(f z, g z, t)$. By Lemma 2.3, we have $f z=g z$

Now, we assume that $w=f z=g z=u z=v z$. Since the pair $(f, v)$ is weakly compatible, $f v z=v f z$ and $f w=$ $f v z=v f z=v w$. Also, since $(u, g)$ is weakly compatible, $g u z=u g z$ and $g w=g u z=u g z=u w$.

We prove that $w=f w$. Using (2) with $x=w, y=z$ for $\alpha=1$, we have

$$
\begin{aligned}
M(f w, u z, k t) \geq & \phi(M(v w, g z, t), M(f w, v w, t), \\
& M(u z, g z, t), M(f w, g z, t), \\
& M(u z, v w, t)), \\
N(f w, u z, k t) \leq & \psi(N(v w, g z, t), N(f w, v w, t), \\
& N(u z, g z, t), N(f w, g z, t), \\
& N(u z, v w, t)) .
\end{aligned}
$$

Since $\phi$ is increasing, and $\psi$ is decreasing in each of its coordinate and $\phi(t, t, t, t, t)>t$ and $\psi(t, t, t, t, t)<t$ for all $t \in[0,1)$, we get $M(f w, w, k t) \geq M(f w, w, t)$ and $N(f w, w, k t) \leq N(f w, w, t)$. By Lemma 2.3, we have $f w=w$. Therefore $w=f w=v w$.

Next, we show that $w=u w$. Using (2) with $x=z$, $y=w$ for $\alpha=1$, we have

$$
\begin{aligned}
& M(f z, u w, k t) \quad \geq \phi(M(v z, g w, t), M(f z, v z, t), \\
& M(u w, g w, t), M(f z, g w, t), \\
& M(u w, v z, t)) \text {, } \\
& N(f z, u w, k t) \quad \leq \psi(N(v z, g w, t), N(f z, v z, t), \\
& N(u w, g w, t), N(f z, g w, t) \text {, } \\
& N(u w, v z, t))
\end{aligned}
$$

and so

$$
\begin{aligned}
M(w, u w, k t) \geq & \phi(M(w, u w, t), M(w, w, t), \\
& M(u w, u w, t), M(w, u w, t), \\
& M(u w, w, t)), \\
N(w, u w, k t) \quad & \psi(N(w, u w, t), N(w, w, t), \\
& N(u w, u w, t), N(w, u w, t), \\
& N(u w, w, t)) .
\end{aligned}
$$


Since $\phi$ is increasing, and $\psi$ is decreasing in each of its coordinate and $\phi(t, t, t, t, t)>t$ and $\psi(t, t, t, t, t)<t$ for all $t \in[0,1)$, we get $M(w, u w, k t) \geq M(w, u w, t)$ and $N(w, u w, k t) \leq N(w, u w, t)$. By Lemma 2.3, we have $w=u w$. Hence $w=u w=g w$. Therefore $w=f w=$ $g w=u w=v w$. That is, $f, g, u$ and $v$ have common fixed point $w \in X$.

Finally, we let $a$ be another common fixed point of mappings $f, g, u$ and $v$. From (2) with $x=w, y=a$ for $\alpha=1$, we have

$$
\begin{aligned}
M(f w, u w, k t) \geq & \phi(M(v w, g a, t), M(f w, v w, t), \\
& M(u a, g a, t), M(f w, g a, t), \\
& M(u a, v w, t)), \\
N(f w, u w, k t) \quad \leq & \psi(N(v w, g a, t), N(f w, v w, t), \\
& N(u a, g a, t), N(f w, g a, t), \\
& N(u a, v w, t)),
\end{aligned}
$$

and

$$
\begin{aligned}
M(w, a, k t) \geq & \phi(M(w, a, t), M(w, w, t), \\
& M(a, a, t), M(w, a, t), \\
& M(a, w, t)), \\
N(w, a, k t) \leq & \psi(N(w, a, t), N(w, w, t), \\
& N(a, a, t), N(w, a, t), \\
& N(a, w, t)),
\end{aligned}
$$

Hence we get $M(w, a, k t) \geq M(w, a, t)$ and $N(w, a, k t) \leq N(w, a, t)$. By Lemma 2.3, we have $w=a$. Therefore $f, g, u$ and $v$ have a unique common fixed point. This completes the proof.

Remark 3.4. Theorem 3.3 improves and generalizes the result of Park[7] without any requirement of containment amongst range sets of the involved mappings and closedness of the subspace.

Corollary 3.5. Let $X$ be an intuitionistic fuzzy metric space, where $*, \diamond$ are continuous t-norm, t-conorm and $f, g$ be mappings from $X$ into itself. Further, let the pair $(f, g)$ is weakly compatible and there exists a constant $k \in\left(0, \frac{1}{2}\right)$ such that

$$
\begin{aligned}
& M(f x, f y, k t) \quad \geq \phi(M(g x, g y, t), M(f x, g x, t) \\
& M(f y, g y, t), M(f x, g y, \alpha t), \\
& M(f y, g x, 2 t-\alpha t)), \\
& N(f x, f y, k t) \leq \psi(N(g x, g y, t), N(f x, g x, t), \\
& N(f y, g y, t), N(f x, g y, \alpha t) \text {, } \\
& N(f y, g x, 2 t-\alpha t))
\end{aligned}
$$

hold for all $x, y \in X, \alpha \in(0,2), t>0$ and $\phi, \psi \in \Phi$. If $(f, g)$ satisfies the (JCLRg) property, then $f, g$ have a unique common fixed point in $X$.
Proof. Taking with $f=u$ and $g=v$ in Theorem 3.3, then we have the result of Corollary 3.5.

\section{References}

[1] S. Chauhan, W. Sintunavarat, P. Kumam, "Common fixed point theorems for weakly compatible mappings in fuzzy metric spaces using (JCLR) property," $A p$ plied Math., vol. 3, pp.976-982, 2012.

[2] A. George, P. Veeramani, "On some results of analysis for fuzzy metric spaces," Fuzzy Sets and Systems, vol. 90, pp. 365-368, 1997.

[3] M. Imdad, J. Ali, "Some common fixed point theorems in fuzzy metric spaces," Math. Commun., vol. 11, pp. 153-163, 2006.

[4] J. Kramosil, J. Michalek, "Fuzzy metric and statistical metric spaces," Kybernetica, vol. 11, pp. 326-334, 1975.

[5] J.S. Park, "On fixed point theorem of weak compatible maps of type $(\gamma)$ in complete intuitionistic fuzzy metric space," Int. J. F. I. S., vol. 11, no. 1, pp. 38-43, 2011.

[6] J.S. Park, "Some fixed point theorem using common property(E.A.) in intuitionistic fuzzy metric space," J. Korean Soc. Math. Educ. Ser. B, vol. 18, no. 3, pp. 255-260, 2011.

[7] J.S. Park, " Fixed point theorem for common property(E.A.) and weak compatible functions in intuitionistic fuzzy metric space," KIIS., vol. 11, no. 3, pp. 149-152, 2011.

[8] J.S. Park, J.H. Park, Y.C. Kwun, “On some results for five mappings using compatibility of type $(\alpha)$ in intuitionistic fuzzy metric space," International J. Fuzzy Logic Intelligent Systems, vol. 8, no. 4, pp. 299-305, 2008.

[9] B. Singh, S. Jain, "Weak compatibility and fixed point theorems in fuzzy metric space," Ganita, vol. 56, pp. 167-176, 2005.

[10] L.A. Zadeh, "Fuzzy sets," Inform. and Control, vol. 8, pp. 338-353, 1965.

\section{Jong Seo Park}

Professor of Chinju National University of Education Research Area: Fuzzy mathematics, Fuzzy fixed point theory, Fuzzy differential equation

E-mail : parkjs@cue.ac.kr 